\title{
Graphene based mobile laboratory in medicine
}

\section{Federica Valentini ${ }^{*}$ and Maurizio Talamo ${ }^{2}$}

${ }^{1}$ Science and Chemical Technologies Department, Tor Vergata University, via della Ricerca Scientifica 1, 00133 Rome, Italy ${ }^{2}$ INUIT Foundation Tor Vergata University, via dell'Archiginnasio snc, 00133 Rome, Italy

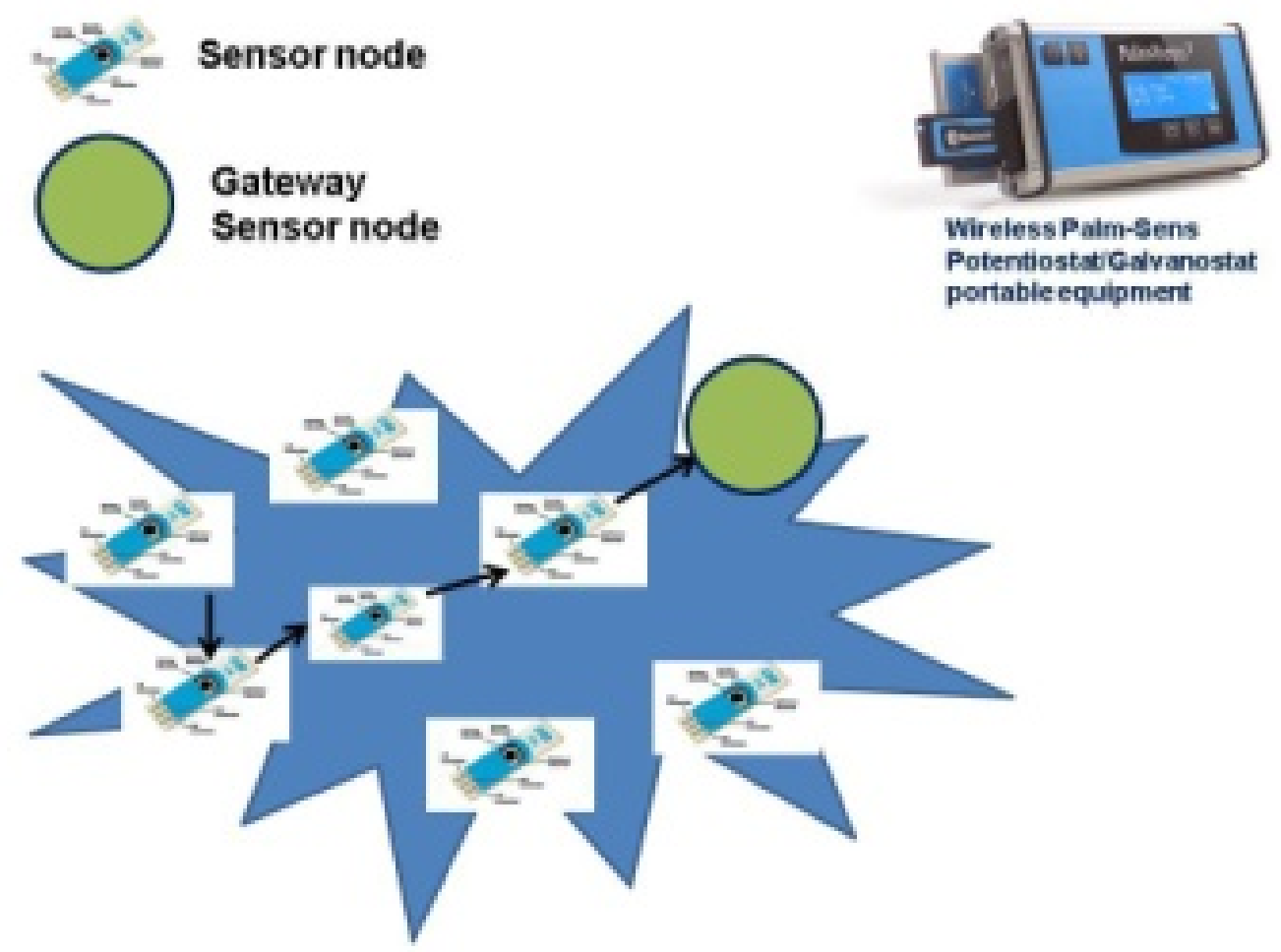

Figure 1. Graphical Abstract

\section{Editorial}

The raise of computing tools and mobile devices improved the quality of clinical research and health care, on a global scale. Wearable electrochemical graphene-based sensors, combined with Smartphone-apps, point-of-need diagnostic tools, and medical-high definition imaging will enable care and enhance the understanding of physiological variability. Furthermore, electrochemical actuators, assembled with functionalized graphene, could provide localized and specific therapies, with a controlled release of specific drugs and therapeutics, in physiological medium. Portable Laboratory prototypes show low sensitivity a high detection of limit (LOD) if compared with the unmovable instrumentations. After the application of graphene, as sensitive layers on sensors and actuators, graphene significantly improves the sensitivity of the movable laboratory. Big data and experimental results can be acquired with portable devices building
${ }^{*}$ Correspondence to: Valentini F, Science and Chemical Technologies Department, Tor Vergata University, via della Ricerca Scientifica 1, 00133 Rome, Italy, E-mail: federica.valentini@uniroma2.it

Special Issue: Nanotechnology: Challenges and Perspectives in Medicine

Dr. Federica Valentini

Department of Sciences and Chemical Technologies

Tor Vergata University

Italy

Maurizio Talamo

Professor

Department of Enterprise Engineering

Italy

Received: September 20, 2018; Accepted: September 24, 2018; Published: September 26, 2018 
a distributed and connected Laboratory with few root nodes. These ones are able to collect and analyse high dimensional big data to refine understanding of molecular loading and release, cytotoxicity checking and cellular uptake, which are critical issues behind above therapies. Recent literature involving data mining and machine learning on data coming from nanotechnologies and nanomedicine, shows the weakness of model of supervised learning and linear or linearized regression. The high number of variables measured, and their heterogeneity are managed via complex linearized models with low quality of results.
Acceptable results in terms of prediction are available only via artificial neural networks (ANN) but these methods often suffer of over fitting and difficult to output causal relationships behind data. We introduced a new causal model based on Kolmogorov complexity and mathematical tools coming from time series. The software tool implementing the model is a causal engine, able to digest high dimensional data and to output causal evidences and risk analysis associated to them. We launched a large set of experiments to stress and try to refine and validate this model (Figure 1).

Copyright: $\odot 2018$ Valentini F. This is an open-access article distributed under the terms of the Creative Commons Attribution License, which permits unrestricted use, distribution, and reproduction in any medium, provided the original author and source are credited. 